\title{
Pancreatoduodenectomía totalmente laparoscópica: Técnica quirúrgica y experiencia inicial*
}

\author{
Drs. NICOLÁS JARUFE C. ${ }^{1}$, JOSÉ IGNACIO FERNÁNDEZ F. ${ }^{1}$, CAMILO BOZA W. ${ }^{1}$, \\ FRANCISCA NAVARRETE C. ${ }^{1}$, ALEX ESCALONA P. ${ }^{1}$, RICARDO FUNKE H. ${ }^{1}$, LUIS IBÁÑEZ A. \\ ${ }^{1}$ Departamento de Cirugía Digestiva. Pontificia Universidad Católica de Chile, Santiago, Chile.
}

\begin{abstract}
Totally laparoscopic pancreaticoduodenectomy: Surgical technique and initial experience
\end{abstract}

Introduction: Nowadays the utility of laparoscopic pancreatic surgery is accepted for a wide variety of indications. However pancreaticoduodenectomy has been considered beyond the possibilities of laparoscopy by the majority of surgeons. The objective of this report is to show our surgical technique and initial experience with totally laparoscopic pancreaticoduodenectomy. Material and Methods: Between november of 2007 and june of 2008, a laparoscopic technique was offered to patients with indication of pancreaticoduodenectomy for different pathologies. We describe the surgical technique, perioperative care, intraoperative complications, need for conversion to open technique, mortality, and early postoperative complications. Results: A total of three patients were subjected to laparoscopic pancreaticoduodenectomy. The surgical indication was an ampullar adenocarcinoma in a 52 years old woman, a pseudopapilar tumor of the pancreatic head in a 17 years old woman, and a duodenal gastrointestinal stromal tumor in a 63 years old man. There was no need for conversion to open technique. There was no mortality. The operative time ranged from 300 to $360 \mathrm{~min}$. The first patient presented an upper gastrointestinal bleeding, from pancreatic anastomoses requiring surgical hemostasis. The last patient developed a self-limited biliary fistula, managed successfully with drains. The hospital stay varied from 8 to 25 days. Discussion: Totally laparoscopic pancreaticoduodenectomy is a feasible procedure, replicating the same steps of open surgery in a reasonable operative time, and with surgical results comparable with open technique.

Key words: Laparoscopy, pancreaticoduodenectomy, Whipple procedure.

\section{Resumen}

Introducción: Actualmente la utilidad de la laparoscopía en la cirugía pancreática es ampliamente aceptada en diversas áreas; sin embargo, la pancreatoduodenectomía había sido considerada un procedimiento fuera de las posibilidades de laparoscopía por la mayoría de los cirujanos. El objetivo de este trabajo es dar a conocer la técnica quirúrgica y nuestra experiencia inicial con la pancreatoduodenectomía totalmente laparoscópica. Material y Métodos: Desde noviembre del 2007 hasta junio del 2008, se ofreció la vía de abordaje laparoscópica a pacientes con indicación de pancreatoduodenectomía curativa por diver-

\footnotetext{
*Recibido el 28 de Agosto de 2008 y aceptado para publicación el 9 de Octubre de 2008.

Correspondencia: Dr. Nicolás Jarufe C.

Marcoleta 352, Santiago, Chile.

Fax: 6329620

E-mail: njarufe@med.puc.cl
} 
sas patologías. Se describe la técnica quirúrgica utilizada, el manejo perioperatorio, complicaciones intraoperatorias, necesidad de conversión a técnica abierta, mortalidad y complicaciones precoces. Resultados: Total $=3$ pacientes. La indicación de cirugía fue por un adenoma ampular en una mujer de 52 años, un tumor pseudopapilar de cabeza de páncreas en una mujer de 17 años y un tumor estromal gastrointestinal duodenal en un hombre de 63 años. No se requirió de conversión a técnica abierta en ningún caso. No hubo mortalidad postoperatoria. Los tiempos operatorios variaron entre 300-360 min. La primera paciente presentó una hemorragia digestiva alta por sangrado del muñón pancreático, requiriendo reoperación. El último paciente presentó una fístula biliar autolimitada, manejada con drenajes. La estadía hospitalaria varió entre 8 y 25 días. Discusión: La pancreatoduodenectomía totalmente laparoscópica es técnicamente factible de realizar, replicando los mismos pasos de la técnica abierta, en un tiempo operatorio razonable, y obteniendo resultados quirúrgicos comparables a la técnica abierta.

Palabras clave: Laparoscopia, pancreatoduodenectomia, operación de Whipple.

\section{Introducción}

Desde la publicación por Whipple en $1935^{1}$, la pancreatoduodenectomía se ha mantenido como el tratamiento estándar de la patología tumoral periampular. Inicialmente este procedimiento implicaba una mortalidad promedio del $30 \%$, la cual se ha reducido en la actualidad a menos de un $5 \%{ }^{2-5}$. Sin embargo, aún mantiene una morbilidad considerable, cercana al $30-40 \%{ }^{6}$.

Desde principios de los años 90, la laparoscopía se ha incorporado progresivamente a una amplia variedad de procedimientos quirúrgicos, con el fin de disminuir el trauma operatorio y la morbilidad derivada de estos. En el caso de la cirugía pancreática, la utilidad de la laparoscopía es actualmente ampliamente aceptada en la etapificación del cáncer de páncreas, enucleación de tumores neuroendocrinos, manejo de complicaciones tardías de la pancreatitis aguda, y resección de tumores del cuerpo y cola ${ }^{7}$. Sin embargo, la pancreatoduodenectomía había sido considerada un procedimiento fuera de las posibilidades de la cirugía laparoscópica por la mayoría de los cirujanos. En 1994 Gagner y Pomp, demuestran por primera vez la factibilidad técnica de la pancreatoduodenectomía por vía laparoscópica $^{8}$. Desde entonces se han publicado sólo pequeñas series de pacientes intervenidos por diversas patologías benignas y malignas, sugiriendo sus resultados que son al menos comparables a los de la técnica abierta ${ }^{9-12}$.

El objetivo de este trabajo es dar a conocer la técnica quirúrgica y nuestra experiencia inicial con la pancreatoduodenectomía realizada totalmente por vía laparoscópica.

\section{Material y Método}

Desde noviembre del 2007 hasta junio del 2008, se ofreció la vía de abordaje laparoscópica a pa- cientes con indicación de pancreatoduodenectomía curativa motivada por diversas patologías, explicando los riesgos y potenciales beneficios del procedimiento.

\section{Técnica quirúrgica}

El paciente es colocado en posición de LloydDavis y Trendelemburg invertido, con el cirujano entre las piernas del paciente y el ayudante a la derecha de este (Figura 1A). Se ingresa a la cavidad peritoneal y se obtiene un neumoperitoneo a $15 \mathrm{mmHg}$ mediante un trocar óptico de $10 \mathrm{~mm}$, insertado $2-5 \mathrm{~cm}$ sobre el ombligo y $2 \mathrm{~cm}$ a la derecha de la línea media.

Se insertan tres trocares adicionales de $10 \mathrm{~mm}$ : uno en el flanco derecho a nivel de la línea axilar anterior, $1-2 \mathrm{~cm}$ bajo el trocar inicial, otro en el flanco izquierdo a nivel de la línea medio clavicular, 1-2 $\mathrm{cm}$ por sobre el trocar inicial, y otro subcostal derecho a nivel de la línea medioclavicular. Se utiliza un separador automático para la retracción hepática a través de un trocar de $5 \mathrm{~mm}$ subxifoídeo (Figura 1B). Se realiza una exploración cuidadosa de toda la cavidad abdominal. Se utilizan como instrumentos de disección el bisturí armónico Ultracision ${ }^{\circledR}$ y gancho. Para la oclusión de vasos sanguíneos de mayor calibre, se utilizaron clips poliméricos Hemo-lok ${ }^{\circledR}$.

Se realiza una maniobra de Kocher extendida (Figura 2A), liberando la totalidad del duodeno hasta el ángulo de Treitz, exponiendo la vena cava y el borde lateral derecho de la aorta mediante bisturí armónico, mientras el ayudante lo tracciona hacia medial con una pinza. Luego se procede a la apertura y sección del ligamento gastrocólico, hasta identificar y seccionar los vasos gastroepiploicos derechos, previa colocación de clip polimérico. Se continúa con la disección a través del ligamento hepatoduodenal y el pedículo hepático con gancho, aislando arterias hepática común, gastroduo- 


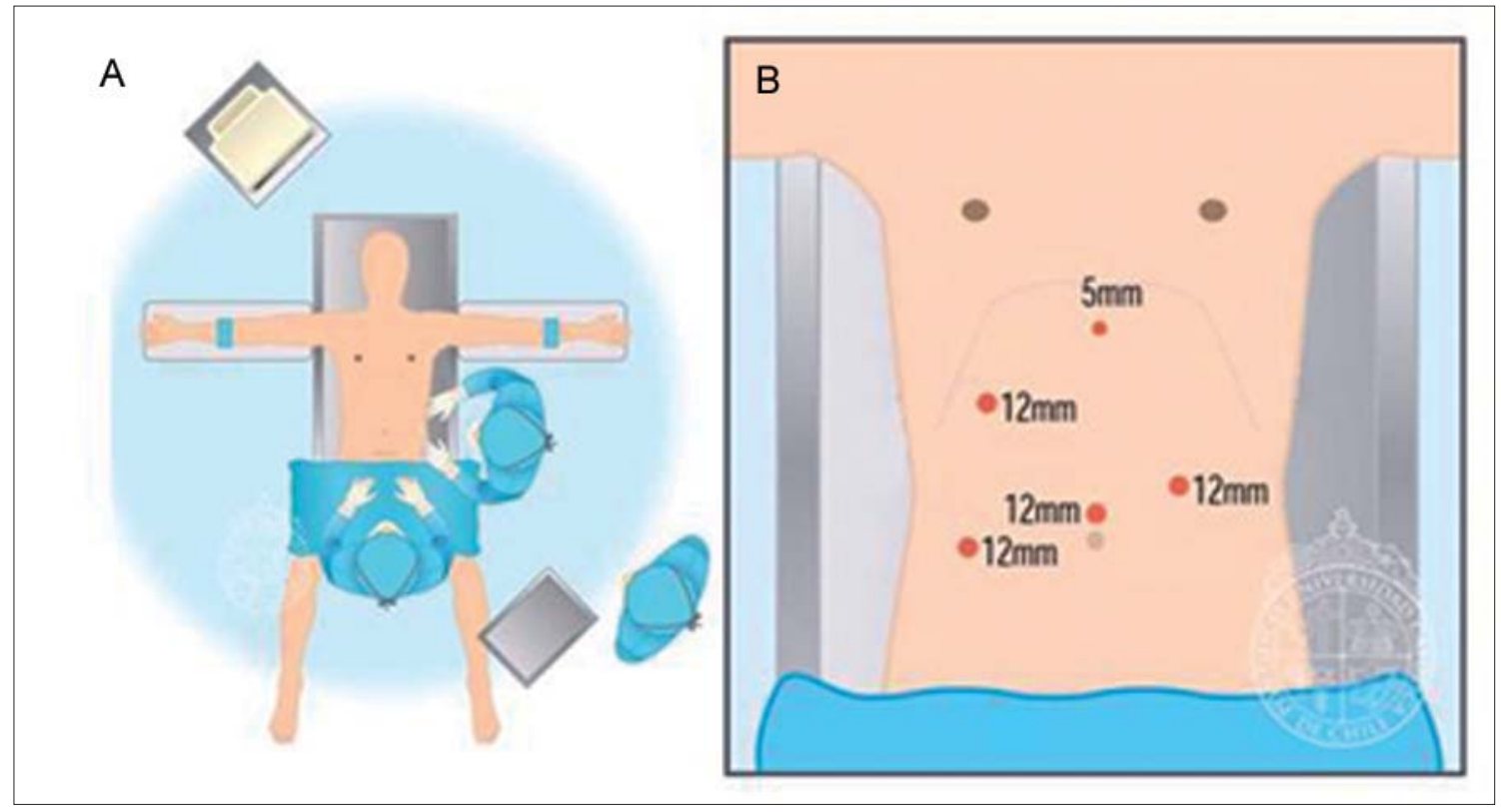

Figura 1. A: Posición del paciente y equipo quirúrgico. B: Sitios de inserción de trocares.

denal, pilórica y hepática propia, para luego disecar y seccionar vía biliar distal, ocluida temporalmente con un clip polimérico.

Se libera el borde superior del duodeno, seccionando la arteria pilórica con bisturí armónico (no se secciona en el caso de preservación pilórica). El estómago es seccionado a nivel del antro con dos cargas azules de stappler gastrointestinal endo-GIA, en el caso de pancreatoduodenectomía tradicional, y se realiza la sección de la primera porción del duodeno $1 \mathrm{~cm}$ distal al píloro, con carga azul en el caso de preservación pilórica. Se continúa con la liberación de la tercera y cuarta porción duodenal del meso-colon transverso. Se secciona el yeyuno a $10 \mathrm{~cm}$ del ángulo de Treitz, con stappler endoGIA carga azul. Se secciona la arteria gastroduodenal, previa colocación de clip polimérico.

Se realiza una disección roma por debajo del cuello pancreático y encima de la vena mesentérica superior (VMS), tallando un túnel con salida suprapancreática. Se rodea el cuello pancreático con una banda elástica, deslizada con ayuda de un separador esofágico (Figura 2B). Posteriormente se realizan unos puntos intracorpóreos de vicryl 3-0 a cada ángulo del cuerpo del páncreas y se secciona con bisturí armónico (Figura 2C). Se aíslan y seccionan las venas tributarias de la cabeza pancreática a la VMS con tijera fría, previa colocación de clips metálicos. El proceso uncinado es adelgazado con bisturí armónico y seccionado utilizando un stappler endo-GIA con carga blanca (Figura 3A).
Se realiza una reconstrucción tipo Child modificada, ascendiendo el yeyuno proximal por vía transmesocólica o antecólica, según cada caso. Se libera el muñón pancreático con gancho, de manera que quede manipulable para la anastomosis. Se realiza una pancreato-gastro anastomosis en un plano, continua, con prolene 3-0, a la cara posterior del estómago en todos los casos (Figura 4B). Se continúa con una hepático-yeyuno-anastomosis con sutura continua en un plano, utilizando PDS 40 o 5-0 (Figura 4A).

En el caso de una pancreatoduodenectomía tradicional, se realiza una gastro-yeyuno anastomosis latero-lateral (Figura 5A), y una duodeno-yeyuno anastomosis termino-lateral en el caso de preservación pilórica (Figura 5B); ambas en dos planos con sutura continua de vicryl 3-0.

Se realiza una colecistectomía con técnica habitual, aseo, hemostasia y cierre de la brecha mesocólica, según corresponda. Se introducen dos drenajes aspirativos Jackson-Pratt, uno subhepático y otro adyacente a la anastomosis pancreática, los que se extraen por los orificios de los trocares de ambos flancos. La pieza quirúrgica se extrae por una incisión de Pfannestiel.

\section{Manejo Postoperatorio}

Los pacientes inician su recuperación en una unidad de cuidado intermedio las primeras 24 horas, para luego ser trasladados a su sala al confir- 


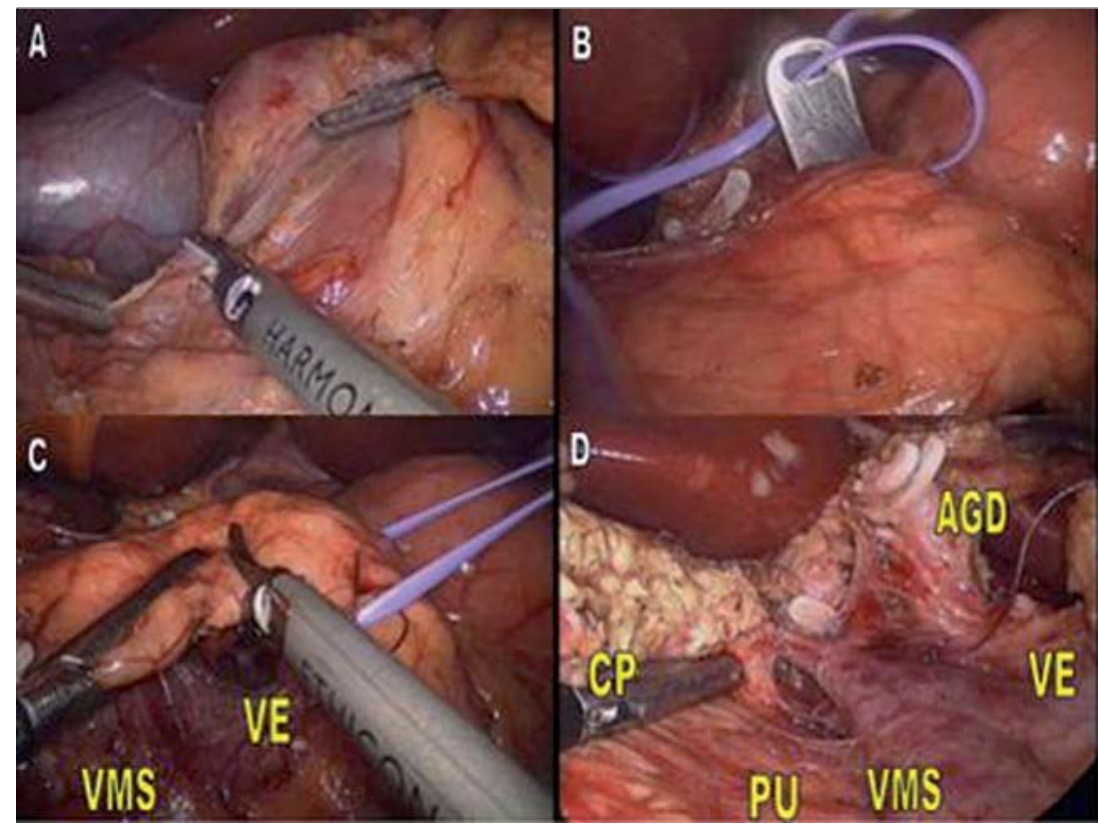

Figura 2. Etapa Resectiva. A: Maniobra de Kocher extendida. B: Disección retropancreática. C: Sección pancreática. D: Cuello pancreático seccionado. $\mathrm{VMS}=$ vena mesentérica superior, $\mathrm{VE}=$ vena esplénica, $\mathrm{AGD}=$ arteria gastroduodenal, $\mathrm{CP}=$ cuello pancreático, $\mathrm{PU}=$ proceso uncinado.

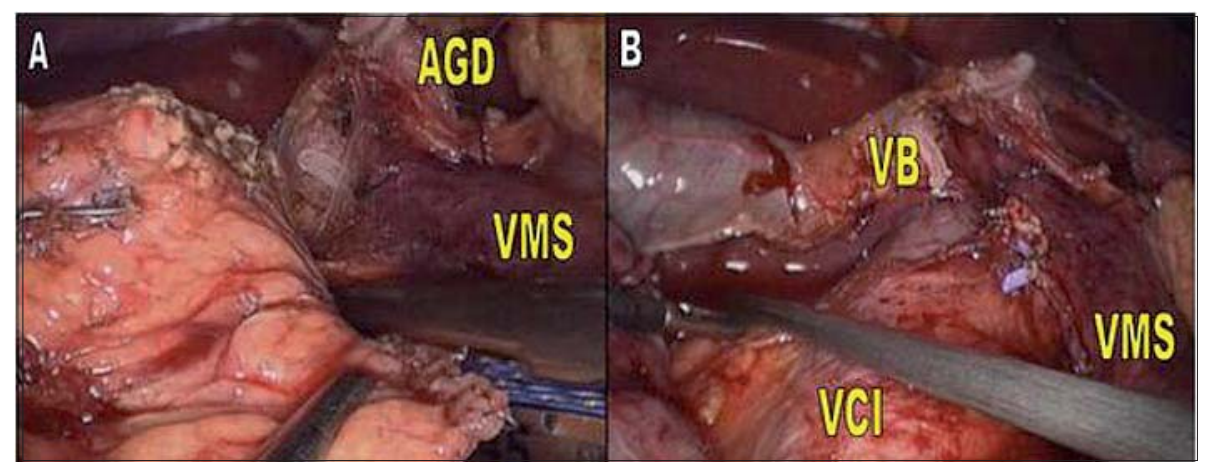

Figura 3. Etapa Resectiva. A: Sección del proceso uncinado con stappler. B: Lecho quirúrgico, una vez completada la resección. $\mathrm{VMS}=$ vena mesentérica superior, $\mathrm{VCI}=$ vena cava inferior, $\mathrm{AGD}=$ arteria gastroduodenal, $\mathrm{VB}=\mathrm{vía} b \mathrm{biliar}$.

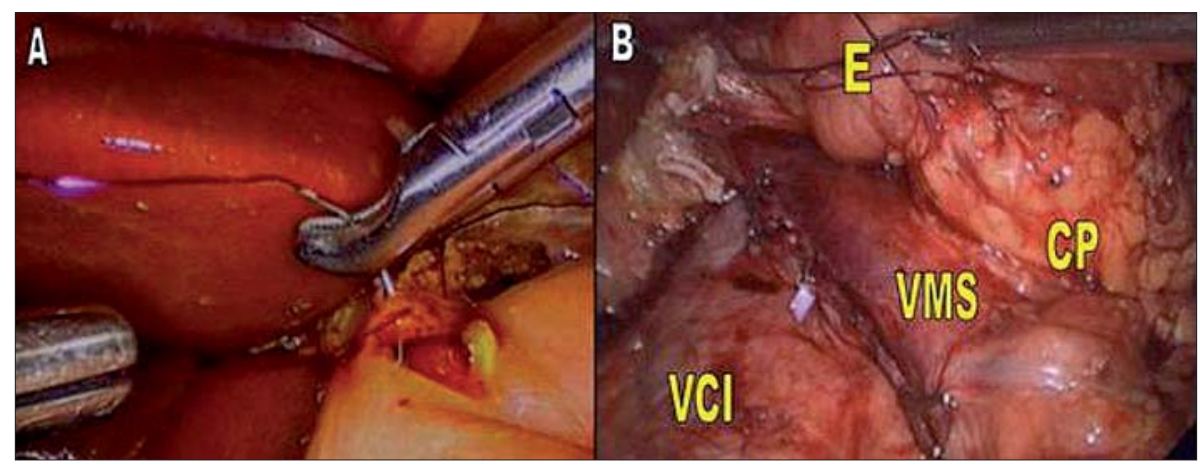

Figura 4. Reconstrucción. A: Hepático-yeyuno anastomosis. B: Pancreato-gastro anastomosis. VMS= vena mesentérica superior, $\mathrm{VCI}=$ vena cava inferior, $\mathrm{CP}=$ cuerpo del páncreas, $\mathrm{E}=$ estómago. 
mar su estabilización. En todos los pacientes se realiza tromboprofilaxis con compresión neumática intra y postoperatoria por 24 horas, para luego continuar con heparina de bajo peso molecular. Se retira el catéter urinario al comprobarse diuresis adecuada, y la sonda la nasogástrica, si esta se mantiene con débitos bajos. Se inicia la realimentación con régimen líquido fraccionado luego de ceder el íleo postoperatorio, progresando a un régimen sólido liviano según tolerancia. Se administra octeotride subcutáneo $100 \mu \mathrm{g}$ cada 12 horas por siete días a todos los pacientes. Los drenajes se retiran según su debito y previo control de amilasa en el líquido al tercer día postoperatorio.

\section{Resultados}

Un total de tres pacientes fueron sometidos a una pancreatoduodenectomía por vía laparoscópica. No se requirió de conversión a técnica abierta en ningún caso. No hubo mortalidad postoperatoria.

El primer caso fue una paciente de sexo femenino y 52 años de edad, con el diagnóstico inicial de un adenoma de la ampolla de Vater. La paciente es intervenida en noviembre del 2007, realizándose una pancreatoduodenectomía sin preservación pilórica, en un tiempo operatorio de 330 minutos. La paciente evoluciona en el postoperatorio inmediato con una hemorragia digestiva alta masiva, debiendo ser reintervenida por vía abierta, encontrándose sangrado del muñón pancreático hacia el estómago, el cual se corrige con sutura de los puntos sangrantes en el parénquima pancreático. En los días posteriores evoluciona con íleo postoperatorio prolongado y mantiene débitos elevados por los drenajes, requiriendo apoyo con nutrición parenteral. El estudio del líquido de los drenajes resulta compatible con linforrea, y descarta fístula pancreática. Posterior a esto la paciente evoluciona en forma favorable, lográndose realimentación progresiva, y es dada de alta a los 25 días postoperatorios. El estudio anatomopatológico finalmente mostró la presencia de un adenocarcinoma tubular bien diferenciado de la ampolla de Vater de $2 \mathrm{~cm}$ de diámetro, que invadía coledoco distal, pared de duodeno y tejido adiposo peripancreático, con bordes quirúrgicos negativos y compromiso de uno de 19 linfonodos resecados. La paciente es derivada para terapia adyuvante.

El segundo caso corresponde a una paciente de sexo femenino y 17 años de edad, con el diagnóstico de una lesión quística en la cabeza del páncreas de $5 \mathrm{~cm}$ de diámetro. Esta lesión provocaba compresión gástrica sintomática y dolor abdominal. En enero del 2008 se realiza una pancreatoduode-

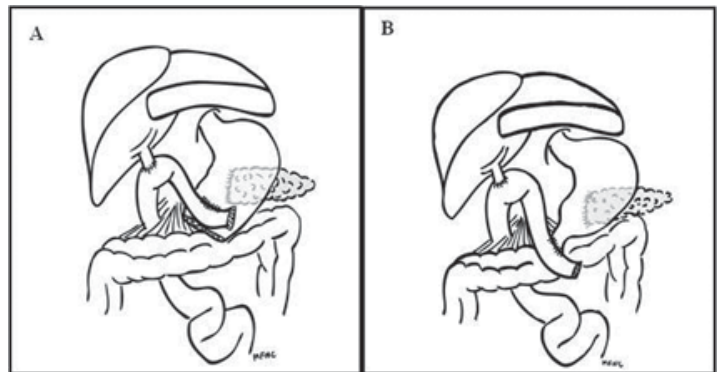

Figura 5. Reconstrucción de pancreatoduodenectomía laparoscópica. A: Sin preservación pilórica. B: Con preservación pilórica.

nectomía con preservación pilórica con un tiempo operatorio de 300 minutos, sin incidentes. Evoluciona favorablemente y sin complicaciones, siendo dada de alta al octavo día postoperatorio. El estudio anatomopatológico, finalmente identifica a la lesión como un tumor pseudopapilar, que se encontraba encapsulado y sin invasión de tejidos adyacentes.

El tercer caso correspondió a un paciente de sexo masculino y 63 años de edad, a quien durante el estudio de un síndrome diarreico crónico y en una enteroclisis por TAC, se le identifica una lesión submucosa del duodeno ulcerada, confirmada por endosonografía. Esta se encontraba $2 \mathrm{~cm}$ proximal a la papila. Se realizó un estudio de etapificación, incluyendo una gamagrafía con Galio-67 pensando en la posibilidad de un carcinoide, el que resulta negativo. Finalmente se decide realizar una pancreatoduodenectomía sin preservación pilórica en junio del 2008, en un tiempo operatorio de 360 minutos. El paciente evoluciona favorablemente los primeros días, presentando filtración biliar que es conducida adecuadamente por los drenajes, y sin repercusión séptica para el paciente. La fístula cede espontáneamente y se le da el alta quirúrgica a los 14 días postoperatorios. El paciente prolonga su hospitalización para continuar el estudio de su síndrome diarreico. El estudio anatomopatológico, identifica a la lesión como un tumor estromal gastrointestinal con bajo índice mitótico.

\section{Discusión}

La pancreatoduodenectomía totalmente laparoscópica es una técnica quirúrgica de alta complejidad, que requiere de experiencia en laparoscopía avanzada y en cirugía hepatobiliar. En esta técnica resulta destacable la excelente visión que se obtiene mediante la visión laparoscópica de cada uno 
de los pasos del procedimiento. Esto se aprecia especialmente durante la disección retropancreática, al separar el cuello del páncreas de la vena mesentérica superior, al obtener una visión incluso mejor que en la técnica abierta. En general, en los tres casos expuestos, la etapa resectiva de la cirugía pudo ser realizada de manera expedita y cómoda. Por otro lado, resulta particularmente compleja la hepático-yeyuno anastomosis en los últimos dos casos, al tratarse de pacientes con vía biliar no dilatada (3-4 mm).

En esta pequeña serie, mostramos una estadía hospitalaria que parece similar a la técnica abierta; sin embargo, se destaca el escaso dolor postoperatorio y la poca repercusión sistémica del trauma quirúrgico.

A pesar de las potenciales ventajas que se esperaría obtener a través de la laparoscopía, los pioneros de la cirugía pancreática laparoscópica parecen estar dudosos acerca del real beneficio de esta vía de abordaje en el caso de la pancreatoduodenectomía ${ }^{13-15}$. Sin embargo, otros autores han presentado experiencias alentadoras, coincidiendo en la necesidad de realizar una adecuada selección de pacientes y contar con un buen entrenamiento del equipo quirúrgico en cirugía hepatobiliar y laparoscopía avanzada, para permitir ofrecer un procedimiento seguro y aprovechar los beneficios de la cirugía mínimamente invasiva ${ }^{12,16}$.

Por el momento las series son pequeñas, y sólo unos pocos cirujanos realizan este procedimiento. Es necesario esperar contar con una mayor experiencia por parte de los grupos que actualmente realizan ésta técnica, y que ésta sea adoptada por otros centros, para poder disponer de resultados que permitan evaluar los reales beneficios de este abordaje.

El presente trabajo es el primer reporte en la literatura médica de pancreatoduodenectomía totalmente laparoscópica en Latinoamérica. En él se demuestra que es un procedimiento técnicamente factible de realizar, replicando los mismos pasos de la técnica abierta, en un tiempo operatorio razonable.

\section{Referencias}

1. Whipple AO, Parsons WB., Mullins CR. Treatment of Carcinoma of the Ampulla of Vater. Ann Surg 1935; 102: 763-779.

2. Trede M, Schwall G, Saeger HD. Survival after pancreatoduodenectomy. 118 consecutive resections without an operative mortality. Ann Surg 1990; 211: 447-458.

3. Cameron JL, Pitt HA, Yeo CJ, Lillemoe KD, Kaufman HS, Coleman J. One hundred and forty-five consecutive pancreaticoduodenectomies without mortality. Ann Surg 1993; 217: 430-435; discussion 435-438.

4. Winter JM, Cameron JL, Campbell KA, Arnold MA, Chang DC, Coleman J, et al. 1423 pancreaticoduodenectomies for pancreatic cancer: A single-institution experience. J Gastrointest Surg 2006; 10: 1199-1210; discussion 1210-1211.

5. Cameron JL, Riall TS, Coleman J, Belcher KA. One thousand consecutive pancreaticoduodenectomies. Ann Surg 2006; 244: 10-15.

6. Turaga K, Kaushik M, Forse RA, Sasson AR. In hospital outcomes after pancreatectomies: An analysis of a national database from 1996 to 2004. J Surg Oncology 2008; 98: 156-160.

7. Melvin WS. Minimally invasive pancreatic surgery. Am J Surg 2003; 186: 274-278.

8. Gagner M, Pomp A. Laparoscopic pylorus-preserving pancreatoduodenectomy. Surg Endosc 1994; 8: 408410.

9. Dulucq JL, Wintringer P, Mahajna A. Laparoscopic pancreaticoduodenectomy for benign and malignant diseases. Surg Endosc 2006; 20: 1045-1050.

10. Pugliese R, Scandroglio I, Sansonna F, Maggioni D, Costanzi A, Citterio D, et al. Laparoscopic pancreaticoduodenectomy: a retrospective review of 19 cases. Surg Laparosc Endosc Percutan Tech 2008; 18: 13-18.

11. Gumbs AA, Gayet B. The laparoscopic duodenopancreatectomy: the posterior approach. Surg Endosc 2008; 22: 539-540.

12. Palanivelu C, Jani K, Senthilnathan P, Parthasarathi R, Rajapandian S, Madhankumar MV. Laparoscopic pancreaticoduodenectomy: technique and outcomes. J Am Coll Surg 2007; 205: 222-230.

13. Uyama I, Ogiwara H, Iida S, Takahara T, Furuta T, Kikuchi K. Laparoscopic minilaparotomy pancreaticoduodenectomy with lymphadenectomy using an abdominal wall-lift method. Surg Laparosc Endosc 1996; 6: 405-410.

14. Cuschieri SA, Jakimowicz JJ. Laparoscopic pancreatic resections. Semin Laparosc Surg 1998; 5: 168-179.

15. Gagner M, Pomp A. Laparoscopic pancreatic resection: Is it worthwhile? J Gastrointest Surg 1997; 1: 20-25; discussion 25-26.

16. Dulucq JL, Wintringer P, Stabilini C, Feryn T, Perissat J, Mahajna A. Are major laparoscopic pancreatic resections worthwhile? A prospective study of 32 patients in a single institution. Surg Endosc 2005; 19: 1028-1034. 